\title{
PRA A*-ALJABAR SEBAGAI SEBUAH POSET
}

\author{
WELLY RAHMAYANTI \\ Program Studi Matematika, \\ Fakultas Matematika dan Ilmu Pengetahuan Alam, Universitas Andalas Padang, \\ Kampus UNAND Limau Manis Padang, Indonesia, \\ welly.rahmayani@gmail.com
}

\begin{abstract}
Abstrak. Sistem matematika $\left(A, \wedge, \vee,(\cdot)^{\sim}\right)$ adalah Pra $\mathrm{A}^{*}$-Aljabar, bila anggotaanggotanya memenuhi sifat-sifat tertentu. Sistem $\left(A, \wedge, \vee,(\cdot)^{\sim}\right)$ ditulis $A$ yang menyatakan Pra $A^{*}$-Aljabar. Misalkan didefinisikan sebuah relasi terurut parsial " $\leqq$ pada Pra A*-Aljabar ( yang anggota-anggotanya memenuhi sifat refleksif, antisimetri, dan transitif). Kemudian $x \leqq y$ jika dan hanya jika $y \wedge x=x \wedge y=x$. Himpunan $A$ bersamasama dengan relasi terurut parsial pada $A$ dinamakan dengan poset. Pada tulisan ini dikaji struktur aljabar dari Pra A*-Aljabar. Selanjutnya, juga dikaji sifat-sifat Pra A*Aljabar sebagai sebuah poset.
\end{abstract}

Kata Kunci: Aljabar Boolean, Poset, Pra A*-Aljabar.

\section{Pendahuluan}

Aljabar Boolean [1] merupakan suatu sistem matematika $\left(B, \wedge, \vee,(\cdot)^{\sim}\right)$ dimana $B$ adalah himpunan tak kosong, $\wedge$ (meet) dan $\vee$ (join) adalah operasi-operasi biner, dan $(\cdot)^{\sim}$ (tilda) adalah operasi tunggal, yang anggota-anggotanya memenuhi sifatsifat: $x \wedge 1=x, x \vee 0=x, x \wedge y=y \wedge x, x \vee y=y \vee x, x \wedge(y \wedge z)=(x \wedge y) \wedge z$, $x \vee(y \vee z)=(x \vee y) \vee z, x \wedge x^{\sim}=0, x \vee x^{\sim}=1, x \wedge x=x, x \vee x=x, x \wedge 0=0$, $x \vee 1=1, x \wedge(x \vee y)=x \vee(x \wedge y)=x, x \wedge(y \vee z)=(x \wedge y) \vee(x \wedge z), x \vee(y \wedge z)=$ $(x \vee y) \wedge(x \vee z),\left(x^{\sim}\right)^{\sim}=x,(x \wedge y)^{\sim}=x^{\sim} \vee y^{\sim},(x \vee y)^{\sim}=x^{\sim} \wedge y^{\sim}$. Gagasan Pra A*-Aljabar pertama kali diperkenalkan pada tahun 2000 oleh J.Venkateswara Rao. Pra $A^{*}$-Aljabar merupakan suatu sistem matematika $\left(A, \wedge, \vee,(\cdot)^{\sim}\right)$ dimana $A$ adalah himpunan tak kosong, $\wedge$ (meet) dan $\vee$ (join) adalah operasi-operasi biner, dan $(\cdot)^{\sim}($ tilda) adalah operasi tunggal, yang anggota-anggotanya memenuhi sifatsifat: $x^{\sim \sim}=x, x \wedge x=x, x \wedge y=y \wedge x,(x \wedge y)^{\sim}=x^{\sim} \vee y^{\sim}, x \wedge(y \wedge z)=(x \wedge y) \wedge z$, $x \wedge(y \vee z)=(x \wedge y) \vee(x \wedge z)$, dan $(x \wedge y)=x \wedge\left(x^{\sim} \vee y\right)$. Misalkan didefinisikan sebuah relasi terurut parsial pada Pra $A^{*}$-Aljabar yaitu " $\leqq "$ ( yang anggota-anggotanya memenuhi sifat refleksif, antisimetri, dan transitif) maka $x \leqq y$ jika dan hanya jika $y \wedge x=x \wedge y=x$. Himpunan $A$ bersama-sama dengan relasi terurut parsial pada $A$ dinamakan dengan poset.

Makalah ini merupakan tinjauan ulang dari rujukan pustaka [3]. Pada makalah ini penulis mengkaji kembali tentang Pra A*-Aljabar sebagai sebuah poset. 


\section{Struktur Aljabar dari Pra A*-Aljabar}

Pada tulisan, akan dikaji struktur aljabar dari Pra A*-Aljabar yang dinyatakan dalam proposisi, lema dan teorema berikut.

Definisi 2.1. [3] Misal A adalah himpunan tak kosong. Suatu sistem matematika $\left(A, \wedge, \vee,(\cdot)^{\sim}\right)$ dikatakan Pra $A^{*}$-Aljabar, dengan $\wedge$ (meet) dan $\vee$ (join) adalah operasi-operasi biner, dan $(\cdot)^{\sim}$ (tilda) adalah operasi tunggal, jika untuk setiap $x, y, z, \in A$, berlaku:
(1) $x^{\sim \sim}=x$
(2) $x \wedge x=x$,
(3) $x \wedge y=y \wedge x$
(4) $(x \wedge y)^{\sim}=x^{\sim} \vee y^{\sim}$,
(5) $x \wedge(y \wedge z)=(x \wedge y) \wedge z$,
(6) $x \wedge(y \vee z)=(x \wedge y) \vee(x \wedge z)$,
(7) $(x \wedge y)=x \wedge\left(x^{\sim} \vee y\right)$.

Definisi 2.2. Misalkan sistem $\left(A, \wedge, \vee,(\cdot)^{\sim}\right)$ adalah Pra $A^{*}$-Aljabar dengan unsur identitas 1 dan 0 . Suatu sistem lain, namakan $A^{*}$ dinamakan dual dari A jika:

(1) $\wedge$ diganti dengan $\vee$,

(2) $\vee$ diganti dengan $\wedge$,

(3) 0 diganti dengan 1 ,

(4) 1 diganti dengan 0 .

Contoh 2.3. Himpunan $\mathbf{W}=\{0,1,2\}$ adalah suatu Pra A*-Aljabar dengan operasi $\vee, \wedge$, dan $(\cdot)^{\sim}$ yang didefinisikan seperti pada tabel berikut:

\begin{tabular}{|l|l|l|l|}
\hline$\wedge$ & 0 & 1 & 2 \\
\hline 0 & 0 & 0 & 2 \\
\hline 1 & 0 & 1 & 2 \\
\hline 2 & 2 & 2 & 2 \\
\hline
\end{tabular}

Tabel 1. Operasi Biner $\wedge$ pada $A$

\begin{tabular}{|c|c|c|c|}
\hline$\vee$ & 0 & 1 & 2 \\
\hline 0 & 0 & 1 & 2 \\
\hline 1 & 1 & 1 & 2 \\
\hline 2 & 2 & 2 & 2 \\
\hline
\end{tabular}

Tabel 2. Operasi Biner $\vee$ pada $A$

Proposisi 2.4. [3] Untuk setiap Pra A*-Aljabar dengan unsur identitas 1, berlaku: 


\begin{tabular}{|c|c|}
\hline$x$ & $x^{\sim}$ \\
\hline 0 & 1 \\
\hline 1 & 0 \\
\hline 2 & 2 \\
\hline
\end{tabular}

Tabel 3. Operasi $\sim$ pada $A$

(1) $x \vee 1=x \vee x^{\sim}$

(2) $x \wedge 0=x \wedge x^{\sim}$, untuk setiap $x \in A$.

Bukti. Ambil $x \in A$.

(1) Akan ditunjukkan $x \vee 1=x \vee x^{\sim}$.

Berdasarkan Definisi 2 mengenai prinsip dualitas dari Pra A*-Aljabar, maka

Definisi 1 (7) dapat ditulis dalam bentuk $x \vee y=x \vee\left(x^{\sim} \wedge y\right)$.

Perhatikan bahwa $x \vee 1=x \vee\left(x^{\sim} \wedge 1\right)=x \vee x^{\sim}$.

(2) Akan ditunjukkan $x \wedge 0=x \wedge x^{\sim}$.

Perhatikan bahwa $x \wedge 0=x \wedge\left(x^{\sim} \vee 0\right)=x \wedge x^{\sim}$

Lema 2.5. [3] Untuk setiap Pra $A^{*}$-Aljabar, berlaku:

(1) $x \vee\left(x^{\sim} \wedge x\right)=x$,

(2) $\left(x \vee x^{\sim}\right) \wedge y=(x \wedge y) \vee\left(x^{\sim} \wedge y\right)$,

(3) $\left(x^{\sim} \vee x\right) \wedge x=x$,

(4) $(x \vee y) \wedge z=(x \wedge z) \vee\left(x^{\sim} \wedge y \wedge z\right)$, untuk setiap $x, y, z \in A$

Bukti. Ambil $x, y, z \in A$.

(1) Akan ditunjukkan $x \vee\left(x^{\sim} \wedge x\right)=x$.

Perhatikan bahwa $x \vee\left(x^{\sim} \wedge x\right)=x \vee x=x$.

(2) Akan ditunjukkan $\left(x \vee x^{\sim}\right) \wedge y=(x \wedge y) \vee\left(x^{\sim} \wedge y\right)$.

Perhatikan bahwa $\left(x \vee x^{\sim}\right) \wedge y=y \wedge\left(x \vee x^{\sim}\right)=(y \wedge x) \vee\left(y \wedge x^{\sim}\right)=$ $(x \wedge y) \vee\left(x^{\sim} \wedge y\right)$

(3) Akan ditunjukkan $\left(x^{\sim} \vee x\right) \wedge x=x$.

Perhatikan bahwa $\left(x^{\sim} \vee x\right) \wedge x=x \wedge\left(x^{\sim} \vee x\right)=x \wedge x=x$.

(4) Akan ditunjukkan $(x \vee y) \wedge z=(x \wedge z) \vee\left(x^{\sim} \wedge y \wedge z\right)$.

Perhatikan bahwa $(x \vee y) \wedge z=z \wedge(x \vee y)=z \wedge\left(x \vee\left(x^{\sim} \wedge y\right)\right)=(z \wedge x) \vee$ $\left(z \wedge x^{\sim} \wedge y\right)=(x \wedge z) \vee\left(x^{\sim} \wedge y \wedge z\right)$

Definisi 2.6. [2] Misalkan $A$ adalah Pra $A^{*}$-Aljabar. Suatu $x \in A$ disebut elemen sentral dari $A$ jika $x \vee x^{\sim}=1$. Selanjutnya, himpunan $\left\{x \in A \mid x \vee x^{\sim}=1\right\}$ adalah himpunan semua elemen sentral dari $A$ yang disebut senter (centre) dari $A$ dan dinotasikan dengan $B(A)$.

Teorema 2.7. [3] Misalkan A adalah Pra $A^{*}$-Aljabar dengan unsur identitas 1, maka $B(A)$ adalah aljabar Boolean dengan operasi $\vee, \wedge,(\cdot) \sim$. 
Bukti. Ambil $x, y, z \in B(A)$. Selanjutnya akan ditunjukkan bahwa Aksioma 1-10 dari Definisi aljabar Boolean terpenuhi.

(1) Akan ditunjukkan $x \vee x^{\sim}=1, x \wedge x^{\sim}=0$.

Berdasarkan Definisi 3, jelas bahwa $x \vee x^{\sim}=1$.

Selanjutnya perhatikan bahwa

$0=1^{\sim}=\left(x \vee x^{\sim}\right)^{\sim}=x^{\sim} \wedge\left(x^{\sim}\right)^{\sim}=x^{\sim} \wedge x$.

(2) Akan ditunjukkan $x \wedge 1=x, x \vee 0=x$.

Perhatikan bahwa $x \wedge 1=x \wedge\left(x \vee x^{\sim}\right)=x \wedge\left(x^{\sim} \vee x\right)=\left(x^{\sim} \vee x\right) \wedge x=x$.

Selanjutnya perhatikan bahwa

$x \vee 0=x \vee\left(x \wedge x^{\sim}\right)=x \vee\left(x^{\sim} \wedge x\right)=x$.

(3) Akan ditunjukkan $x \wedge 0=0, x \vee 1=1$.

Perhatikan bahwa $x \wedge 0=x \wedge\left(x \wedge x^{\sim}\right)=(x \wedge x) \wedge x^{\sim}=x \wedge x^{\sim}=0$.

Selanjutnya perhatikan bahwa

$x \vee 1=x \vee\left(x \vee x^{\sim}\right)=(x \vee x) \vee x^{\sim}=x \vee x^{\sim}=1$.

(4) Akan ditunjukkan $x \wedge(x \vee y)=x \vee(x \wedge y)=x$

Perhatikan bahwa $x \wedge(x \vee y)=(x \vee 0) \wedge(x \vee y)=x \vee(0 \wedge y)=x \vee 0$.

Sedangkan untuk $x \vee(x \wedge y)=x$ adalah dual dari $x \wedge(x \vee y)=x$.

Karena $A$ adalah Pra $A^{*}$-Aljabar dan berdasarkan prinsip dualitas maka jelas bahwa Definisi aljabar Boolean (Aksioma 2, 3, 5, 8, 9 dan 10) terpenuhi.

Jadi karena $B(A)$ memenuhi Aksioma 1-10 dari Definisi aljabar Boolean maka $B(A)$ adalah aljabar Boolean.

Lema 2.8. [3] Misalkan $A$ adalah Pra $A^{*}$-Aljabar dengan unsur identitas 1, maka berlaku:

(1) jika $x, y \in B(A)$ maka $x \wedge x^{\sim} \wedge y=x \wedge x^{\sim}$,

(2) $x \wedge(x \vee y)=x \vee(x \wedge y)=x$ jika dan hanya jika $x, y \in B(A)$.

Bukti. Misal $A$ adalah Pra A*-Aljabar,

(1) Jika $x, y \in B(A)$, akan ditunjukkan $x \wedge x^{\sim} \wedge y=x \wedge x^{\sim}$.

Ambil $x, y \in B(A)$ maka berdasarkan Definisi 3 berlaku $x \vee x^{\sim}=1$,

$x \wedge x^{\sim}=0$ dan $y \vee y^{\sim}=1, y \wedge y^{\sim}=0$.

Perhatikan bahwa: $x \wedge x^{\sim} \wedge y=\left(x \wedge x^{\sim}\right) \wedge y=0 \wedge y=y \wedge 0=0=x \wedge x^{\sim}$.

(2) Akan ditunjukkan bahwa $x \wedge(x \vee y)=x \vee(x \wedge y)=x$ jika dan hanya jika $x, y \in B(A)$.

Berdasarkan Teorema 1 maka $B(A)$ adalah aljabar Boolean. Berarti memenuhi Definisi $1(7)$, artinya $x, y \in B(A)$. Dan sebaliknya, jika $x, y \in B(A)$ maka jelas berlaku $x \wedge(x \vee y)=x \vee(x \wedge y)=x$.

\section{Struktur Aljabar dari Pra A*-Aljabar}

Pada tulisan, akan dibahas sifat-sifat Pra A*-Aljabar sebagai sebuah poset yang disampaikan dalam beberapa definisi, teorema, dan lema berikut.

Definisi 3.1. [3] Misalkan A adalah Pra A*-Aljabar. Definisikan" $\leqq "$ pada A dengan $x \leqq y$ jika dan hanya jika $y \wedge x=x \wedge y=x$. 
Lema 3.2. [3] Jika $A$ adalah sebuah Pra $A^{*}$-Aljabar, maka $(A, \leqq)$ adalah sebuah poset.

Bukti. Misalkan $A$ adalah Pra $\mathrm{A}^{*}$-Aljabar, akan dibuktikan bahwa $(A, \leqq)$ adalah sebuah poset yaitu akan ditunjukkan bahwa relasi " $\leqq$ " adalah refleksif, antisimentri dan transitif,

(1) Ambil $x \in A$. Akan ditunjukkan $x \leqq x, \forall x \in A$.

Karena $A$ adalah Pra $\mathrm{A}^{*}$-Aljabar. Artinya $x \wedge x=x$ maka $x \leqq x, \forall x \in A$. Jadi relasi " $\leqq$ adalah refleksif.

(2) Ambil $x, y \in A$. Misalkan $x \leqq y$ dan $y \leqq x$. Akan ditunjukkan bahwa $x=y$. Berdasarkan Definisi 4, berlaku $y \wedge x=x \wedge y=x$ dan $x \wedge y=y \wedge x=y$.

Perhatikan bahwa: $x=x \wedge y=y \wedge x=y$. Jadi terbukti bahwa " $\leqq$ adalah antisimetri.

(3) Ambil $x, y, z \in A$. Misalkan $x \leqq y$ dan $y \leqq z$. Akan ditunjukkan $x \leqq z$. Berdasarkan Definisi 4, berlaku $y \wedge x=x \wedge y=x$ dan $z \wedge y=y \wedge z=y$.

Perhatikan bahwa: $x=x \wedge y=x \wedge(y \wedge z)=(x \wedge y) \wedge z=x \wedge z$. Berdasarkan Definisi 4 berlaku bahwa jika $z \wedge x=x \wedge z=x$ maka $x \leqq z$. Jadi terbukti bahwa " $\leqq$ adalah transitif.

Dari 1 sampai dengan 3 terbukti bahwa $(A, \leqq)$ adalah poset.

Teorema 3.3. [3] Dalam poset $(A, \leqq)$, untuk sebarang $x \in A$, berlaku: sup $\left\{x, x^{\sim}\right\}=x \vee x^{\sim}$ dan inf $\left\{x, x^{\sim}\right\}=x \wedge x^{\sim}$.

Bukti. Misalkan $(A, \leqq)$ adalah poset, dan ambil $x \in A$ sebarang.

(1) Akan ditunjukkan $\sup \left\{x, x^{\sim}\right\}=x \vee x^{\sim}$.

Pada Lema 1 (3), $\forall x \in A$ berlaku $x \wedge\left(x \vee x^{\sim}\right)=x$ dan $x^{\sim} \wedge\left(x \vee x^{\sim}\right)=x^{\sim}$. Akibatnya dengan menggunakan Definisi 4 diperoleh $x \leqq x \vee x^{\sim}$ dan $x^{\sim} \leqq$ $x \vee x^{\sim}$. Karena itu $x \vee x^{\sim}$ adalah batas atas dari $\left\{x, x^{\sim}\right\}$.

Misalkan $n \in A$ adalah batas atas lain dari $\left\{x, x^{\sim}\right\}$, maka $x \leqq n$ dan $x^{\sim} \leqq n$. Berdasarkan Definisi 4 diperoleh $n \wedge x=x \wedge n=x$ dan $n \wedge x^{\sim}=x^{\sim} \wedge n=x^{\sim}$.

Perhatikan bahwa

$\left(x \vee x^{\sim}\right) \wedge n=n \wedge\left(x \vee x^{\sim}\right)=(n \wedge x) \vee\left(n \wedge x^{\sim}\right)=(x \wedge n) \vee\left(x^{\sim} \wedge n\right)=x \vee x^{\sim}$.

Ini menunjukkan bahwa $x \vee x^{\sim} \leqq n$, sehingga $x \vee x^{\sim}$ adalah batas atas terkecil dari $\left\{x, x^{\sim}\right\}$. Jadi $\sup \left\{x, x^{\sim}\right\}=x \vee x^{\sim}$.

(2) Akan ditunjukkan $\inf \left\{x, x^{\sim}\right\}=x \wedge x^{\sim}$.

Perhatikan bahwa $x \wedge\left(x \wedge x^{\sim}\right)=x \wedge x^{\sim}$ dan $\left(x \wedge x^{\sim}\right) \wedge x^{\sim}=x \wedge x^{\sim}$. Akibatnya dengan menggunakan Definisi 4 diperoleh $x \wedge x^{\sim} \leqq x$ dan $x \wedge x^{\sim} \leqq x^{\sim}$. Karena itu $x \wedge x^{\sim}$ adalah batas bawah dari $\left\{x, x^{\sim}\right\}$.

Misalkan $m \in A$ adalah batas bawah lain dari $\left\{x, x^{\sim}\right\}$, maka $m \leqq x$ dan $m \leqq x^{\sim}$. Berdasarkan Definisi 4 diperoleh $x \wedge m=m \wedge x=m$ dan $x^{\sim} \wedge m=$ $m \wedge x^{\sim}=m$.

Perhatikan bahwa

$m \wedge\left(x \wedge x^{\sim}\right)=(m \wedge x) \wedge x^{\sim}=m \wedge x^{\sim}=m$. 
Ini menunjukkan bahwa $m \leqq x \wedge x^{\sim}$, sehingga $x \wedge x^{\sim}$ adalah batas bawah terbesar dari $\left\{x, x^{\sim}\right\}$. Jadi inf $\left\{x, x^{\sim}\right\}=x \wedge x^{\sim}$.

Teorema 3.4. [3] Dalam poset $(A, \leqq)$ dengan unsur identitas 1 , untuk sebarang $x, y \in A$ berlaku inf $\{x, y\}=x \wedge y$.

Bukti. Akan ditunjukkan $\inf \{x, y\}=x \wedge y$.

Perhatikan bahwa $(x \wedge y) \wedge x=x \wedge y$ dan $(x \wedge y) \wedge y=x \wedge y$. Akibatnya dengan menggunakan Definisi 4 diperoleh $x \wedge y \leqq x$ dan $x \wedge y \leqq y$. Karena itu $x \wedge y$ adalah batas bawah $\{x, y\}$.

Misalkan $m \in A$ adalah batas bawah lain dari $\{x, y\}$, maka $m \leqq x$ dan $m \leqq y$. Berdasarkan Definisi 4 diperoleh $x \wedge m=m \wedge x=m$ dan $y \wedge m=m \wedge y=m$. Perhatikan bahwa

$m \wedge(x \wedge y)=(m \wedge x) \wedge y=m \wedge y=m$.

Ini menunjukkan bahwa $m \leqq x \wedge y$, sehingga $x \wedge y$ adalah batas bawah terbesar dari $\{x, y\}$. Jadi $\inf \{x, y\}=x \wedge y$.

Teorema 3.5. [3] Dalam poset $(A, \leqq)$ dengan unsur identitas 1 , untuk sebarang $x, y \in B(A)$ maka $\sup \{x, y\}=x \vee y$.

Bukti. Misalkan $(A, \leqq)$ adalah poset. Pada Lema $2(2), \forall x, y \in B(A)$ berlaku $(x \vee y) \wedge x=x \wedge(x \vee y)=x$ dan $(x \vee y) \wedge y=y \wedge(x \vee y)=y$. Akibatnya dengan menggunakan Definisi 4 diperoleh $x \leqq x \vee y$ dan $y \leqq x \vee y$. Karena itu $x \vee y$ adalah batas atas dari $\{x, y\}$.

Misalkan $z$ adalah batas atas lain dari $\{x, y\}$, maka $x \leqq z$ dan $y \leqq z$. Berdasarkan Definisi 4 diperoleh $z \wedge x=x \wedge z=x$ dan $z \wedge y=y \wedge z=y$. Perhatikan bahwa

$(x \vee y) \wedge z=z \wedge(x \vee y)=(z \wedge x) \vee(z \wedge y)=(x \wedge z) \vee(y \wedge z)=x \vee y$.

Ini menunjukkan bahwa $x \vee y \leqq z$, sehingga $x \vee y$ adalah batas atas terkecil dari $\{x, y\}$. Jadi $\sup \{x, y\}=x \vee y$.

Teorema 3.6. [3] Dalam poset $(A, \leqq)$, jika $x, y \in B(A)$ maka $x \vee y \leqq x \vee x^{\sim}$.

Bukti. Misalkan $(A, \leqq)$ adalah poset. Ambil $x, y \in B(A)$. Akan ditunjukkan $x \vee y \leqq$ $x \vee x^{\sim}$.

Perhatikan bahwa

$\left(x \vee x^{\sim}\right) \wedge(x \vee y)=(x \vee y) \wedge\left(x \vee x^{\sim}\right)=((x \vee y) \wedge x) \vee\left((x \vee y) \wedge x^{\sim}\right)=$ $(x \wedge(x \vee y)) \vee\left(x^{\sim} \wedge(x \vee y)\right)=x \vee\left(x^{\sim} \wedge(x \vee y)\right)=x \vee\left(x^{\sim} \wedge y\right)=x \vee y$.

Maka berdasarkan Definisi 4 diperoleh $x \vee y \leqq x \vee x^{\sim}, x, y \in B(A)$.

Teorema 3.7. [3] Dalam poset $(A, \leqq)$, jika $x \leqq y$ maka untuk sebarang $z \in A$, berlaku:

(1) $z \wedge x \leqq z \wedge y$,

(2) $z \vee x \leqq z \vee y$. 
Bukti. Misalkan $(A, \leqq)$ adalah poset, dan jika $x \leqq y$ maka $y \wedge x=x \wedge y=x$. Ambil $z \in A$.

(1) Akan ditunjukkan $z \wedge x \leqq z \wedge y$.

Perhatikan bahwa

$(z \wedge y) \wedge(z \wedge x)=(z \wedge x) \wedge(z \wedge y)=((z \wedge x) \wedge z) \wedge y=((x \wedge z) \wedge z) \wedge y=$ $(x \wedge(z \wedge z)) \wedge y=(x \wedge z) \wedge y=(z \wedge x) \wedge y=z \wedge(x \wedge y)=z \wedge x$.

Maka berdasarkan Definisi 4 diperoleh $z \wedge x \leqq z \wedge y$.

(2) Akan ditunjukkan $z \vee x \leqq z \vee y$.

Perhatikan bahwa

$(z \vee y) \wedge(z \vee x)=(z \vee x) \wedge(z \vee y)=z \vee(x \wedge y)=z \vee x$

Maka berdasarkan Definisi 4 diperoleh $z \vee x \leqq z \vee y$.

\section{Kesimpulan}

Misal suatu sistem matematika $\left(A, \wedge, \vee,(\cdot)^{\sim}\right)$ adalah Pra $A^{*}$-Aljabar. Selanjutnya diperoleh struktur aljabar dari Pra $A^{*}$-Aljabar seperti pada bagian 2. Dengan mendefinisikan sebuah relasi terurut parsial pada Pra A*-Aljabar maka himpunan $A$ bersama-sama dengan relasi terurut parsial pada $A$ dinamakan dengan poset. Kemudian diperoleh sifat-sifat Pra A*-Aljabar sebagai sebuah poset seperti pada bagian 3 .

\section{Ucapan Terima kasih}

Penulis mengucapkan terima kasih kepada Bapak Admi Nazra, Ibu Nova Noliza Bakar, Ibu Lyra Yulianti, Bapak Dodi Devianto, Bapak Efendi, yang telah memberikan masukan dan saran sehingga makalah ini dapat diselesaikan dengan baik.

\section{Daftar Pustaka}

[1] Birkhoff, G. 1973. Lattice Theory,Third Edition. American Mathematical Society.

[2] Satyanarayana, A. dan J.V. Rao. 2011. Representation of Pre A*-Algebra by Section of Sheaves. International Journal of computational Cognition, Vol. 9, No. 2, June 2011 (40-44).

[3] Rao, J.V. dan S. Rao. 2009. Pre A*-Algebra as a Poset. African Journal of mathematics and computer Science Research, Vol 2 (4), May 2009 (073-080). 